\title{
Dispositif en ligne d'entraînement à la résolution de problèmes de physique
}

\author{
Pierre-Xavier Marique \\ Université de Liège, didactique de la physique \\ pxmarique@ulg.ac.be \\ Maud Jacquet \\ Université de Liège, LabSET \\ maud.jacquet@ulg.ac.be \\ François Georges \\ Université de Liège, LabSET \\ fr.georges@ulg.ac.be \\ Maryse Hoebeke \\ Université de Liège, didactique de la physique \\ m.hoebeke@ulg.ac.be \\ Marianne Poumay \\ Université de Liège, LabSET \\ m.poumay@ulg.ac.be
}

Dans le cadre de la lutte contre l'échec en premier cycle supérieur, le LabSET et des enseignants de physique de l'université de Liège ont développé un dispositif en ligne d'entraînement à la résolution de problèmes en physique. Deux problématiques sont abordées ici : le diagnostic de la maîtrise des processus cognitifs à mobiliser pour résoudre des problèmes de physique et le lien entre entraînement en ligne et performance des étudiants lors de la résolution de problèmes à l'examen. Les analyses reposent sur une étude menée auprès de 876 étudiants inscrits en première année en médecine. Elles ont été effectuées sur la base des données subjectives (auto-évaluation du processus et du produit) et objectives (nombre de connexions aux exercices en ligne, résultats de maîtrise des questions spécifiques aux processus cognitifs étudiés, taux de réussite et notes aux examens). Les résultats indiquent que le processus d'analyse est celui posant le plus de difficultés aux étudiants. De plus, une dépendance est observée entre travail en ligne et réussite aux problèmes présentés à l'examen de juin. Les notes des étudiants ayant travaillé en ligne sont supérieures à celles des étudiants n'ayant réalisé aucun problème en ligne. Cependant, étant donné que les étudiants ayant réussi l'examen de physique sont aussi ceux ayant réussi les épreuves dans les autres matières scientifiques, il est difficile d'établir un rapport de causalité entre travail en ligne et performances.

: télé-apprentissage, résolution de problèmes, diagnostic, entraînement à une tâche, processus cognitif

\section{Online training platform for resolution of problems in physics}

As part of projects aiming to reduce failure during bachelor studies, LabSET and physics teachers of the university of Liège developed an online package designed to improve skills in solving physics problems. Research focuses, on one hand, on diagnosis as to whether cognitive processes used in solving physics problems are mastered by the user or not and, on the other hand, on the link between effective online training and students performance at solving examination problems. Analyses rely on a 
study conducted with 876 first-year medicine students. They were performed on the basis of subjective (self-evaluation of process and product) and objective (number of connections, results on specific questions for each cognitive process, passing rate and examination results) data. At the end of the research, we observed that the analysis process is the one for which the students show most difficulties. Almost $50 \%$ of the students are aware of this. Although the strength of association is weak to medium, dependence is observed between online work and success at solving the examination problems. The results of students who worked online are higher than those of students who did not. However, considering that students who passed the physics examination also passed the examinations on other scientific subjects, it is difficult to establish a causality link between online work and performance.

: e-learning, problem solving, diagnosis, task training, cognitive process

\section{Introduction et problématique}

Les établissements d'enseignement supérieur de la Fédération Wallonie-Bruxelles de Belgique constatent, depuis plusieurs années, des échecs répétés dans les matières scientifiques comme la physique, la biologie ou encore la chimie. En physique, quelle que soit la filière d'étude, les notes obtenues par les étudiants sont en effet majoritairement basses (moyennes inférieures à la note de passage de 10/20) et contribuent à leur échec en fin d'année. Selon les enseignants, dans de nombreux cas, c'est principalement la note obtenue aux résolutions de problèmes qui provoque l'échec de l'étudiant.

Partant de ce constat, une collaboration entre le laboratoire de soutien à l'enseignement par la télématique (LabSET) et le département de physique de l'université de Liège tente via un projet innovant, déployé par l'Académie Wallonie-Europe, de comprendre pourquoi les étudiants échouent aux examens de physique, et particulièrement à la partie consacrée aux résolutions de problèmes. Ont-ils un problème de méthode de résolution ? À quel niveau de la résolution d'un problème de physique se situent les difficultés rencontrées par les étudiants ? Les échecs sont-ils dus à un manque d'entraînement? Que mettre en œuvre pour les aider efficacement ?

Deux axes de travail ont été choisis. Le premier doit permettre l'identification du processus cognitif et de l'étape de résolution posant le plus de difficultés lors d'une résolution de problème. En effet, il est crucial d'identifier le processus cognitif insuffisamment ou inefficacement mobilisé pour mener à bien une résolution de problème, afin de permettre au corps enseignant d'établir des pistes de travail en lien avec les besoins des étudiants et ainsi favoriser la réussite.

Le second doit servir à fournir aux étudiants un outil leur permettant d'améliorer leurs performances en résolution de problèmes de physique. Ce dispositif d'entraînement doit donc proposer des résolutions décomposées afin de permettre à l'étudiant de travailler chacune des parties d'une résolution et de pouvoir identifier quelles sont les étapes mal ou non maîtrisées. Chacune des étapes doit être finalisée par la présence d'un feed-back permettant à l'étudiant d'ajuster sa résolution.

Après avoir rappelé le contexte dans lequel nous évoluons et le cadre théorique sur lequel nous nous appuyons, nous présenterons le dispositif mis en place au cours de cette collaboration. Par la suite, nous présenterons une expérimentation réalisée avec un large public de première année universitaire. Nous tenterons alors de répondre aux deux questions : quels sont le processus cognitif et l'étape de résolution les moins bien maîtrisés ? Y a-t-il un impact de l'entraînement en ligne sur la réussite?

\section{Contexte et dispositif}

\subsection{Le contexte}

Les différentes institutions universitaires proposent plusieurs types d'activités ayant pour but d'aider les étudiants de première année. Nous pouvons par exemple citer des tests formatifs, des séances collectives d'exercices, des séances de remédiation en groupes ou des séances individuelles à propos des méthodes de travail. Le but de notre démarche est d'ajouter à ces dispositifs une ressource disponible à tout moment et n'importe où, moyennant une connexion Internet, et fournissant à l'étudiant les traces de ses propres raisonnements, l'aidant ainsi à s'autoévaluer.

En particulier, notre démarche se concentre sur la difficulté des étudiants à résoudre des problèmes. Newell et Simon (1972) considèrent qu'une personne rencontre un problème lorsqu'elle veut quelque chose et ne sait pas par quel moyen y parvenir. Dans le domaine de l'enseignement, Tardif précise qu'un problème existe « quand une personne, étant donné la base de connaissances dans sa mémoire à long terme, ne peut immédiatement trouver la suite des opérateurs pour parvenir à l'état désiré en tenant compte des contraintes et des données initiales. » (Tardif, 1992, 
p. 230). Comme Jacquet et al. le soulignent, «cette nécessité de faire des hypothèses et de recourir à des opérations intermédiaires pour rechercher la (ou les) solution(s) différencie le problème d'une simple question d'application, explicite quant à elle et dans laquelle toutes les données sont disponibles. Résoudre un problème est donc un processus complexe. La difficulté éprouvée en ce domaine par les étudiants participe grandement à leur échec dans une discipline comme la physique qui a fait de ce processus l'un des déterminants essentiels du passage des étudiants dans l'année supérieure. » (Jacquet et al., 2012).

Leclercq, Laszlo et De Landsheere (1978) tentent d'expliquer les difficultés qu'ont les étudiants à résoudre des problèmes. Ils avancent notamment le manque d'esprit critique des étudiants, qui questionnent trop peu la vraisemblance des énoncés, leur difficulté à organiser les éléments qui leur sont donnés et à hiérarchiser les informations. Ils dénoncent le fait qu'ils utilisent peu leur imagination pour faire des hypothèses basées sur l'analogie, que leurs raisonnements logiques sont trop peu systématiques, incomplets ou mal assurés, que leurs estimations sont trop rares et peu pertinentes alors que leurs erreurs de mesure sont très larges. Ils relèvent aussi qu'ils doutent de leur capacité à surmonter la situation seuls, qu'ils renoncent trop vite et démissionnent à tort, peu conscients de l'étendue réelle de leurs capacités, peu entraînées et peu valorisées dans ce mode de travail au sein de nos formations.

\subsection{Le dispositif}

Nous avons opté pour un dispositif en ligne afin d'aider les étudiants à appréhender la résolution de problèmes et lutter contre le manque d'entraînement. Ce type d'outil présente l'avantage d'être accessible en permanence et n'importe où, moyennant une connexion Internet.

Le dispositif en ligne a été conçu pour entraîner l'apprenant à comprendre les consignes, à les analyser et à identifier les informations utiles à la mobilisation et la combinaison ad hoc d'outils nécessaires à la résolution de problèmes. Il offre aux apprenants des feed-back pour leur permettre d'ajuster leur démarche. Au terme de l'exercice, il les outille pour analyser leur performance. L'outil proposé n'a pas comme objectif de fournir directement une remédiation mais bien d'aider l'étudiant et l'équipe enseignante à diagnostiquer précisément les lacunes afin de mettre en place une remédiation efficace. Il doit notamment permettre de répondre aux questions: comprennent-ils correctement l'énoncé d'un problème ? Savent-ils identifier les données pertinentes ou choisir le modèle à appliquer adapté à la situation donnée?

Le dispositif sert donc d'outil d'entraînement aux étudiants en proposant un nombre important de problèmes variés à résoudre et de laboratoire de recherche à l'équipe pédagogique.

Le dispositif a été développé sur la plateforme d'apprentissage en ligne Blackboard 9.1 mise à disposition par l'université de Liège (ULg) pour tous les étudiants et enseignants de l'université. Cette plateforme contient de nombreuses fonctionnalités : conception d'évaluations automatisées, mise à disposition de ressources de formats variés, outils d'échanges privés et publics entre enseignants et étudiants, outils de travail collaboratif, etc.

Deux méthodes d'accompagnement à la résolution de problèmes ont été mises en place dans le dispositif en ligne : la résolution guidée et la résolution libre.

La résolution guidée est une méthode constituée d'un énoncé de problème et de dix questions guidant l'étudiant vers la résolution. Huit de ces dix questions ont été établies en référence aux processus cognitifs impliqués dans la résolution de problèmes. En s'appuyant sur la littérature liée aux processus cognitifs, notamment les travaux de Bloom (1956) et ceux d'Anderson et al. (2001), ainsi que sur les témoignages d'acteurs de terrain issus du département de physique (professeurs académiques et assistants), une décomposition d'une résolution type de problèmes de physique rencontrés en première année à l'université a été développée et mise en œuvre. Trois processus cognitifs principaux mobilisés dans la résolution de problèmes ont été identifiés :

- la compréhension, à l'œuvre lors de la lecture de l'énoncé et permettant l'identification de l'objet de la demande ; - l'analyse, à l'origine de l'identification des données pertinentes dans l'énoncé et de la sélection des modèles, vus au cours, utiles à la résolution du problème spécifique défini dans l'énoncé, et permettant aussi une mise en lien des éléments et une planification des étapes de résolution ;

- l'application permettant la mise en pratique des concepts en référence au problème donné.

La mobilisation correcte de ces trois processus, appliquée à un énoncé donné, ouvre sur une solution unique. Pour qu'une résolution soit réussie, ces trois processus doivent être mobilisés et combinés efficacement. Un manque de compréhension implique nécessairement un échec de l'analyse. De même, un échec de l'analyse ne permettra pas aux étudiants d'appliquer aux données les formules et modèles adéquats pour résoudre le problème. Réussir une résolution implique donc une maîtrise de ces trois processus et la mobilisation appropriée de ceux-ci.

Comme le montre le tableau 1 , une question est associée à la compréhension, quatre portent sur l'analyse et trois sur l'application. Pour avancer dans la résolution, l'étudiant doit répondre à chacune des questions. Le format de 
question utilisé varie (QCM, appariement, QRM, etc.) mais permet systématiquement une notation directe (ne nécessitant pas l'intervention de l'enseignant). Une note sur 100 est attribuée une fois le problème soumis.

\begin{tabular}{|c|c|c|c|}
\hline Bloc & $\begin{array}{l}\mathrm{N}^{\circ} \\
\mathrm{Q}\end{array}$ & Étape & Question \\
\hline Compréhension & 1 & $\begin{array}{l}\text { Compréhension de } \\
\text { l'énoncé }\end{array}$ & $\begin{array}{l}\text { Avant de résoudre le problème il } \\
\text { est nécessaire de réfléchir à }(\mathrm{au}) \ldots \\
\text { Parmi les propositions ci-dessous, sélectionnez } \\
\text { celles qui nous aideront à résoudre le problème. }\end{array}$ \\
\hline \multirow[t]{4}{*}{ Analyse } & 2 & $\begin{array}{l}\text { Chemin } \quad \mathrm{de} \\
\text { résolution }\end{array}$ & $\begin{array}{l}\text { Parmi les propositions ci-dessous, sélectionnez et } \\
\text { placez dans l'ordre les étapes nécessaires et } \\
\text { correctes pour la résolution du problème. }\end{array}$ \\
\hline & 3 & $\begin{array}{lr}\text { Détermination du } \\
\text { modèle } \\
\text { appliquer }\end{array}$ & $\begin{array}{l}\text { Sélectionnez, parmi les modèles proposés ci- } \\
\text { dessous, tous ceux (un ou plusieurs) qui vont } \\
\text { fournir les relations à utiliser pour calculer la } \\
\text { réponse à la question posée. }\end{array}$ \\
\hline & 4 & $\begin{array}{l}\text { Détermination des } \\
\text { formules à utiliser }\end{array}$ & $\begin{array}{l}\text { Les étapes à réaliser pour résoudre le problème } \\
\text { peuvent nécessiter l'utilisation de formules issues } \\
\text { de votre cours de physique. Parmi la liste de } \\
\text { formules ci-dessous, nous vous demandons } \\
\text { d'identifier celle(s) utile(s) pour résoudre en tout } \\
\text { ou en partie le problème. }\end{array}$ \\
\hline & 5 & $\begin{array}{l}\text { Détermination des } \\
\text { informations utiles }\end{array}$ & $\begin{array}{l}\text { Parmi les informations ci-dessous, sélectionnées } \\
\text { dans l'énoncé, cochez uniquement celles qui } \\
\text { seront nécessaires pour effectuer l'ensemble des } \\
\text { calcules. }\end{array}$ \\
\hline \multirow[t]{3}{*}{ Application } & 6 & $\begin{array}{lr}\text { Application } & \text { des } \\
\text { formules à } & \text { la } \\
\text { situation } & \end{array}$ & $\begin{array}{l}\text { Parmi les propositions suivantes, sélectionnez les } \\
\text { expressions permettant de résoudre le problème } \\
\text { (en accord avec le système d'axes choisis } \\
\text { précédemment). }\end{array}$ \\
\hline & 7 & $\begin{array}{l}\text { Réponse } \\
\text { numérique } \\
\text { problème }\end{array}$ & Choisissez la bonne réponse. \\
\hline & 8 & $\begin{array}{l}\text { Unité de la } \\
\text { réponse }\end{array}$ & $\begin{array}{l}\text { Donnez le symbole de l'unité de la réponse } \\
\text { demandée. Le symbole sera donné... }\end{array}$ \\
\hline
\end{tabular}

\section{Tableau 1: questions définies en référence aux processus cognitifs impliqués dans une résolution de problèmes}

À ces huit questions sont associées deux questions métacognitives incitant les étudiants à évaluer leur travail. La première porte sur le produit, à savoir, l'étudiant pense-t-il que sa réponse est adéquate et la seconde, sur le processus, à savoir quelles sont les étapes qui lui ont posé problème. Ces questions ont pour objectif d'inciter les étudiants à prendre du recul pour se situer par rapport à l'objectif visé. Évaluer leurs productions leur permettra de réguler leur façon d'apprendre et en conséquence, d'ajuster leurs actions pour progresser, l'objectif étant d'augmenter l'efficacité des apprentissages (Zimmerman, 2002).

Voici le canevas final de résolution d'un problème : 


\section{Quatre blocs - Dix questions}

\begin{tabular}{|c|c|}
\hline Compréhension & 1. Compréhension de l'énoncé \\
\hline Analyse & $\begin{array}{l}\text { 2. Chemin de résolution } \\
\text { 3. Détermination du modèle à appliquer } \\
\text { 4. Détermination des formules à utiliser } \\
\text { 5. Détermination des informations utiles }\end{array}$ \\
\hline Application & $\begin{array}{l}\text { 6. Application des formules à la situation } \\
\text { 7. Réponse numérique au problème } \\
\text { 8. Unité de la réponse }\end{array}$ \\
\hline Métacognition & $\begin{array}{l}\text { 9. Réflexion sur le produit } \\
\text { 10. Réflexion sur le processus }\end{array}$ \\
\hline
\end{tabular}

Figure 1 : le canevas de résolution guidée

Nous nous sommes également fortement inspirés des travaux portant sur le retour d'information vers l'étudiant, communément nommé feed-back. Nicol considère l'évaluation et son corollaire, le feed-back, comme des éléments essentiels à l'autorégulation de l'étudiant et à son apprentissage et en précise les contours (Nicol, 2009a, 2009b). En effet, dans le but d'améliorer leurs performances et d'augmenter leur motivation, il est important de fournir aux étudiants des feed-back plus nombreux et de plus grande valeur diagnostique. Dans notre cas, vu les grands nombres d'étudiants présents dans la population concernée, il était impossible d'envisager des feed-back individuels fournis par l'équipe pédagogique. Or, un feed-back se doit d'être individualisé au maximum. C'est pourquoi, à nouveau, le choix d'un dispositif en ligne est adéquat, car il permet de multiplier, enrichir et personnaliser les feed-back présentés. À l'issue de chaque question, un feed-back immédiat est présenté. Ils peuvent contenir du texte, des images et des animations. Ces feed-back sont fournis à l'étudiant qu'il ait répondu correctement à la question ou non. Dans le premier cas, cela le confortera dans sa résolution et lui permettra de s'appuyer sur la démarche mise en place pour arriver au résultat. Dans le second cas, il obtiendra des informations précises sur le chemin qu'il convenait de suivre. Il pourra alors persévérer et adapter sa résolution, ce qui serait impossible sans feed-back immédiat.

En résumé, les problèmes de résolution guidée permettent donc aux étudiants d'être accompagnés dans la résolution d'un problème. La décomposition en sous-questions soutient l'identification du ou des processus cognitif(s) posant le plus de difficultés aux étudiants. En permettant une progression malgré de potentielles erreurs, toutes les étapes de la résolution peuvent être entraînées et l'ensemble des processus cognitifs peut être abordé. La présence de feed-back permet aux étudiants d'identifier leurs erreurs et de se corriger pour avancer dans la résolution.

Actuellement, plus de 70 problèmes de résolution guidée ont été élaborés et mis en ligne. Dans le but de préparer progressivement les étudiants à la résolution de problèmes telle que rencontrée en condition d'examen, la résolution libre est proposée en complément de la résolution guidée. La méthode de résolution libre est plus complexe pour l'étudiant: seuls l'énoncé du problème et une demande de solution lui sont présentés. Une question de réflexion portant sur la cohérence de sa réponse (métacognition) est ensuite posée. En plus de mettre l'étudiant en situation proche de l'examen, la résolution libre a pour objectif de lui permettre de prendre du recul par rapport à son apprentissage et d'essayer d'aborder seul la résolution de problèmes. Il doit planifier seul les différentes actions à mener et identifier par lui-même ses difficultés.

Après la soumission de sa solution, l'étudiant bénéficie d'un feed-back complet lui permettant d'évaluer ses actions et d'ajuster son raisonnement le cas échéant dans la résolution de problèmes futurs. Ce feed-back sous forme audiovisuelle réalisé sur tablette graphique ou par l'intermédiaire d'un logiciel d'animation présente une résolution de problème réalisée «en direct» par un enseignant. Lors de cette résolution, il veille à faire les liens avec les différents processus cognitifs sollicités. L'ordre des étapes de la solution fournie dans le feed-back multimédia correspond aux huit étapes du processus de résolution guidée. L'assistant prend alors soin d'expliciter chacune des 
phases de la résolution et de faire intervenir les termes «compréhension», «analyse » et «application» correspondant aux trois processus cognitifs rencontrés ainsi que les termes associés aux huit étapes de résolution. Ce déroulement en temps réel permet d'insister sur la séquence de résolution, le chemin à suivre permettant d'aller de l'énoncé à la solution. Une attention particulière a donc été portée à l'aspect didactique des feed-back. En proposant des films réalisés sur tablette montrant un assistant en train de résoudre le problème, la mise en place d'un apprentissage vicariant est visée (Bandura, 1980), permettant à l'étudiant d'apprendre en voyant quelqu'un d'autre faire.

Plus de 30 énoncés de résolution libre ont été créés et mis en ligne.

La partie suivante décrit le contexte d'utilisation de ce dispositif de formation en ligne ainsi que les modalités de récolte et d'analyse de données pour répondre aux questionnements relatifs à la portée diagnostique de l'outil et à son efficacité.

\section{Méthodologie}

Le dispositif en ligne est intégré dans le cours de physique intitulé « Bases physiques et mathématiques des sciences biomédicales » dispensé en première année de médecine et dentisterie à l'ULg. Il s'agit d'un des cours les plus importants du cursus de première année; il représente 11 crédits sur un total de 60 pour l'ensemble des cours de l'année académique. Seuls les autres cours de sciences fondamentales (biologie et chimie) affichent un nombre de crédits équivalent ou supérieur.

Ce cours est composé de quatre parties: 65 heures de cours théoriques données de manière ex cathedra en amphithéâtre, 24 heures de travaux pratiques en laboratoire, 20 heures de séances d'exercices et problèmes réparties en dix séances données par les assistants du cours aux étudiants répartis en "petits» groupes, 20 heures de remédiation données sous la forme de permanences se tenant quatre demi-journées par semaine.

Dans le cadre de cette expérimentation, seuls six problèmes portant sur la thématique des fluides étaient proposés aux étudiants (trois problèmes de « résolution guidée » et trois problèmes de « résolution libre ») afin de garder une cohérence avec la matière vue en cours à ce moment de l'année. Ce dispositif a été disponible durant une période précise : entre le 29 mars 2012 et la fin de la seconde session (mi-septembre). Cependant, les données analysées ici ne concernent que le travail effectué en ligne par les étudiants avant l'examen de physique de la session des mois de mai et juin. L'utilisation du dispositif est facultative, bien que des conditions aient à chaque fois été mises en place pour encourager les étudiants à utiliser le dispositif. L'utilisation du dispositif se fait en marge du cours ; en effet, cette ressource est à la disposition des étudiants mais l'enseignant n'intègre pas le dispositif dans sa pratique en se servant, par exemple, de certains problèmes comme base d'un cours.

L'examen de juin est composé des éléments suivants: un problème de mécanique des fluides, un problème d'électricité, un questionnaire composé de questions à choix multiples et portant sur la théorie, une partie laboratoire. Le dispositif proposé en ligne est vaste et peut être à l'origine de nombreuses questions de recherche. Deux questions principales ont été ciblées dans cette étude.

Premièrement, quel est le processus cognitif posant le plus de difficultés aux étudiants lors de la résolution de problèmes? Est-ce la compréhension du problème, l'analyse, l'application ou encore une combinaison de plusieurs processus qui est à l'origine de leurs mauvaises performances? La perception qu'ont les étudiants de ce qui leur pose des difficultés sera aussi abordée. Sont-ils conscients de ce qui est le moins bien réussi ? Cette adéquation entre difficulté réelle et difficulté perçue est essentielle pour remédier aux faiblesses des étudiants puisque, sans connaissance de ces difficultés, la mise en place de méthodes efficaces pour y remédier est peu probable.

Ensuite, la question d'un lien entre travail en ligne et performances à l'examen est abordée. Les étudiants ayant travaillé en ligne montrent-ils un taux de réussite plus élevé ou de meilleures notes à la résolution de problèmes proposés lors de l'examen que ceux n'ayant pas travaillé en ligne ? Si le dispositif est efficace, une réponse positive devrait être apportée à cette question. Afin d'établir cet éventuel lien entre travail en ligne et performances à l'examen, les résultats des étudiants dans les autres matières scientifiques seront analysés. En effet, avoir travaillé avec le dispositif proposé ne devrait pas avoir d'influence sur les performances dans les autres matières que la physique. Si un lien entre travail en ligne et performances à l'examen des autres matières est constaté, des caractéristiques propres à l'étudiant dans sa réussite comme sa capacité d'assimilation, son niveau de départ ou encore son engagement dans la tâche, devront être mises en avant, et ce au détriment du rôle du dispositif.

\section{1. Échantillon}

Sur les 980 étudiants inscrits en première année de médecine et dentisterie à l'université de Liège, seuls les 876 étudiants ayant présenté l'examen de physique lors de la session de juin 2012 constituent notre échantillon. 


\subsection{Procédures de recueil et analyse des données}

Pour vérifier nos hypothèses, nous avons procédé à des analyses quantitatives et qualitatives.

En ce qui concerne la question portant sur les processus cognitifs posant le plus de difficultés aux étudiants, les scores des étudiants aux différentes questions de compréhension, d'analyse et d'application des problèmes de résolution guidée serviront de base à notre analyse. Les réponses à ces questions et les scores associés sont automatiquement enregistrés dans la plateforme d'apprentissage en ligne. Toutes les tentatives, considérées comme valides, effectuées par les étudiants, quel que soit leur nombre, seront ici considérées. De plus, les réponses données aux questions de métacognition seront utilisées pour appréhender la perception qu'ont les étudiants de leurs difficultés.

De nombreuses réponses de type "sans réponse» ont été observées dans les tentatives des étudiants. Pour s'approcher au plus près d'une représentation réelle, un nettoyage du tableau de données a été effectué. Les principes qui ont présidé à ce nettoyage sont repris ci-dessous. Étant donné qu'au niveau de la résolution de problèmes, le processus est séquentiel et se doit d'être continu, ne pas répondre à des questions du bloc «Analyse » (bloc $\mathrm{n}^{\circ} 2$ ) a comme nécessaire conséquence que la réflexion sur les questions suivantes de type «Application » (bloc $n^{\circ} 3$ ) ne peut être menée à bien. Nous avons donc décidé de ne pas considérer les tentatives des étudiants ayant omis de répondre à $50 \%$ ou plus des questions portant sur l'analyse (bloc $\mathrm{n}^{\circ} 2$ ). D'ailleurs, majoritairement, ces étudiants n'ont fourni aucune réponse aux questions du bloc «Application » (bloc n ${ }^{\circ} 3$ ). Au final, les étudiants n'ont droit qu'à une réponse omise dans le bloc $\mathrm{n}^{\circ} 2$ pour que la tentative soit considérée.

Les tentatives des étudiants ayant donné moins de 2 réponses sur 3 au bloc $n^{\circ} 3$ ont été éliminées en raison d'un soupçon d'abandon. Les tentatives restantes sont considérées comme valides.

En ce qui concerne la mise en évidence d'un lien entre travail en ligne et performances, notre analyse sera nourrie des éléments suivants. Tout d'abord, deux populations d'étudiants, extraites de notre échantillon, seront considérées : les étudiants ayant réalisé les six problèmes proposés en ligne (étudiants reconnus sous l'appellation « ayant travaillé en ligne »), et les étudiants n'ayant réalisé aucun problème en ligne (reconnus sous l'appellation «n'ayant pas travaillé en ligne »). Ensuite, étant donné que le dispositif en ligne n'entraine que la résolution de problèmes et ne comporte pas d'élément théorique, seuls les résultats d'examen portant sur la résolution de problèmes (notes et taux de réussite) seront considérés. Aucune hypothèse quant à l'impact du travail en ligne sur les composantes théoriques de l'examen n'est formulée et cette dimension ne sera pas étudiée.

Enfin, les notes et les taux de réussite obtenus dans les autres matières scientifiques (biologie et chimie) seront utilisés pour déterminer si le lien entre travail en ligne et réussite à l'examen ne peut être attribuable à d'autres facteurs que celui de réaliser des problèmes en ligne. Par une analyse descriptive, les deux questions suivantes seront posées :

- les étudiants ayant réussi l'examen de physique ont-ils aussi réussi les autres matières scientifiques, à savoir la biologie et la chimie?

- Y-a-t-il une différence de médianes en biologie et en chimie entre les étudiants ayant travaillé en ligne et ceux ne l'ayant pas fait?

Si les réponses sont positives, le rôle du dispositif d'aide dans la réussite des étudiants ne pourra être mis en avant puisque ce dispositif n'a a priori pas les caractéristiques permettant d'affecter le travail en biologie ou en chimie. En effet, le dispositif d'aide à la réussite permet d'améliorer la mobilisation et la réalisation des processus cognitifs impliqués dans la résolution de problèmes de physique, mais pas les caractéristiques propres aux autres matières considérées. Si les réponses sont négatives, le rôle du dispositif en ligne dans la réussite de ces étudiants en physique pourra alors être mis en avant.

\subsection{Méthode d'analyse des données}

Une analyse descriptive des réponses données aux différentes questions de compréhension, d'analyse et d'application permettra d'identifier le processus posant le plus de difficultés. Si les étudiants sont aptes à utiliser efficacement un processus impliqué dans la résolution de problèmes, alors des réponses justes devraient être apportées aux questions se rapportant à ce processus.

De la même manière, la confrontation entre les difficultés ressenties par les étudiants et les difficultés détectées par le système sera possible en analysant de façon descriptive les réponses des étudiants dans les différents problèmes. La dernière question portant sur la ou les étapes ayant posé le plus de difficultés permet d'obtenir des indications sur la difficulté ressentie.

La question d'un lien potentiel entre travail en ligne et performances à l'examen pourra être étudiée en confrontant les taux de réussite et les notes à la partie de l'examen de juin portant sur la résolution de problèmes et les traces de 
fréquentation du système. Si le dispositif aide les étudiants dans leur apprentissage, nous devrions constater de meilleures performances en résolution de problèmes (taux de réussite, notes) pour ceux ayant travaillé en ligne (soit ayant réalisé les six problèmes proposés) par rapport aux étudiants n'ayant pas réalisé de problème en ligne.

Pour mettre en évidence un éventuel lien entre travail en ligne et réussite à la résolution de problèmes à l'examen, le test de dépendance $\chi^{2}$ de Pearson sera utilisé. Ce test indique s'il y a une dépendance entre les deux variables (par exemple : réalisation de problèmes en ligne et résultats à l'examen) mais ne dit rien sur la force d'association entre les variables. En cas de dépendance, la force d'association entre ces deux variables devra par la suite être analysée afin d'en déterminer le degré de dépendance. Pour cela, les coefficients d'association suivants seront calculés : le V de Cramer, le coefficient de contingence ou encore le $\mathrm{T}$ de Tschuprow. La force d'association s'exprime en pourcentage, représentant le rapport entre la dépendance calculée et la dépendance statistique complète. Plus le pourcentage est élevé, plus la force d'association est importante. En plus de la force d'association, la modalité de variables participant le plus à cette dépendance sera examinée. Pour cela, la contribution de chaque modalité de variables sur la réussite ou l'échec sera déterminée. Dans le cas d'une indépendance totale, chaque modalité présente une contribution de $25 \%$. Dans les autres cas, plus la contribution est faible, moins la modalité contribue à la dépendance, et, plus la contribution est élevée et donc distante de $25 \%$, plus elle contribue à la dépendance.

Le test des médianes sera également appliqué entre les deux populations précitées sur les notes à la résolution de problèmes à l'examen ainsi que celles dans les autres matières scientifiques. Ce test a pour but de déterminer la médiane de l'ensemble des observations et de dénombrer, dans chacune des populations étudiées, les observations inférieures et les observations supérieures à cette médiane. Le choix de ce test non paramétrique tient au fait de l'absence de normalité des distributions dans les deux groupes.

\subsection{Considérations éthiques}

Estimant a priori l'entraînement potentiellement bénéfique pour tous, aucun groupe contrôle n'a été établi. Tous les étudiants ont bénéficié d'une liberté d'accès aux ressources en ligne. Certains ont choisi de s'y investir fortement, d'autres moins et d'autres encore ont préféré ne pas l'utiliser.

Par ailleurs, l'ensemble des données sont traitées de manière anonyme.

\section{Résultats}

\subsection{Quels sont le processus cognitif et l'étape de résolution les moins bien maîtrisés?}

En analysant les réponses fournies par les étudiants aux différentes questions des différentes tentatives valides et ce, sur les trois problèmes de résolution guidée, le processus cognitif posant le plus de difficultés dans la résolution pourra être identifié de façon objective. De plus, en analysant les réponses données par les étudiants à la dernière question de métacognition, les difficultés perçues par les étudiants seront abordées. Une incohérence entre difficulté réelle et difficulté perçue pourrait être une des causes de leur souci à résoudre correctement un problème complexe. En effet, sans connaissance de ses faiblesses, il n'est pas possible d'y remédier.

Voici le nombre total de tentatives valides réalisées par problème :

\begin{tabular}{|l|l|}
\hline Problème & Nombre de tentatives \\
\hline Problème 1 & 348 \\
\hline Problème 2 & 217 \\
\hline Problème 3 & 225 \\
\hline
\end{tabular}

\section{Tableau 2 : nombre de tentatives considérées pour chaque problème de résolution guidée}

Pour le problème 1, le bloc impliquant la compréhension a été correctement réalisé pour 199 tentatives (soit 57,2 \%). L'analyse nécessaire à la résolution du problème (bloc $\mathrm{n}^{\circ} 2$ ) a été correctement réalisée pour 17 tentatives uniquement (soit 4,9\%). Les questions d'application ont été correctement complétées pour 89 tentatives (soit $25,6 \%)$.

\begin{tabular}{|l|l|l|l|}
\hline & \multicolumn{3}{l|}{ Processus cognitif } \\
\hline & Compréhension & Analyse & Application \\
\hline Problème 1 (\%) & 57,2 & 4,9 & 25,6 \\
\hline
\end{tabular}




\begin{tabular}{|l|l|l|l|}
\hline Problème 2 (\%) & 78,3 & 49,23 & 52,1 \\
\hline Problème 3 (\%) & 65,8 & 2,2 & 39,1 \\
\hline
\end{tabular}

\section{Tableau 3 : pourcentage de réussite aux différents processus cognitifs dans les trois problèmes de résolution guidée}

Pour le problème 2 également, l'analyse est le processus le moins bien maîtrisé. Remarquons cependant que le pourcentage de réussite dans le bloc analyse $(49,3 \%)$ est plus élevé que dans le cas des autres problèmes. Le pourcentage de réussite dans ce bloc est proche du pourcentage de réussite au bloc application $(52,1 \%)$. De plus, le pourcentage global de réussite est meilleur, et cela se marque aussi dans chacun des trois processus impliqués.

La tendance est la même pour le problème 3 que pour le premier: le processus de compréhension est celui qui semble être le plus maîtrisé $(65,8 \%)$ à l'inverse de l'analyse qui est un domaine dans lequel les étudiants semblent avoir beaucoup de difficultés $(2,2 \%)$. Le bloc « application » est réussi à 39,1\% par les étudiants.

En analysant spécifiquement les questions pour les problèmes 1 et 3, ce sont les questions 2 et 5 qui sont les moins bien réussies. Ces questions font partie du bloc «analyse». Elles portent sur le chemin de résolution et la détermination des informations utiles. Pour le problème 2, qui encore une fois se distingue des deux autres, la question la moins bien réussie est la question de la détermination des formules (question 4). Dans tous les cas, nous nous situons ici dans des questions portant sur l'analyse du problème.

\begin{tabular}{|l|l|l|l|l|}
\hline Pourcentage de réussite & Problème 1 & Problème 2 & Problème 3 \\
\hline Bloc & Question & 67,8 & 83,4 & 76 \\
\hline Compréhension & Compréhension de l'énoncé & 6,6 & 51,6 & 1,3 \\
\hline Analyse & Mise en ordre du chemin de résolution & & & \\
& & & & \\
& & & & \\
\cline { 3 - 6 } & & & & \\
\cline { 2 - 6 } & Détermination de(s) modèle(s) à appliquer & 9,8 & 59 & 14,7 \\
\cline { 2 - 6 } & Détermination de(s) formules à utiliser & 20,4 & 44,7 & 52,9 \\
\cline { 2 - 6 } & Détermination des informations utiles & 6,6 & 56,7 & 8,9 \\
\hline \multirow{5}{*}{ Application } & Application des formules à la situation & 9,5 & 48,4 & 20,4 \\
\cline { 2 - 6 } & Réponse numérique & 32,5 & 42,4 & 44 \\
\hline & Unités de la réponse & 52,3 & 88,9 & 72 \\
\hline
\end{tabular}

\section{Tableau 4 : Pourcentage de réussite aux différentes questions dans les trois problèmes de résolution guidée}

Les étudiants perçoivent-ils que leurs difficultés se situent dans l'analyse des données du problème ? En analysant les réponses apportées aux deux dernières questions de chaque problème ${ }^{1}$, il est constaté que la perception des étudiants est congruente avec leurs performances. Pour les problèmes 1 et 3 , les questions portant sur l'étape de mise en ordre du chemin de résolution et sur le choix du modèle à appliquer sont sélectionnées par une proportion importante de participants (respectivement entre $47,3 \%$ et $51,8 \%$ et entre $29,3 \%$ et $37,5 \%$ des étudiants ayant répondu à ces questions). Ces deux étapes font partie du bloc analyse. Pour le problème 2, la question portant sur la mise en ordre du chemin de résolution est aussi sélectionnée par une proportion non négligeable d'étudiants (environ $27 \%$ ). La question portant sur la réponse numérique (bloc «Application») apparaît aussi comme problématique pour plus de $40 \%$ des étudiants.

Globalement, moins de questions sont identifiées comme problématiques par les étudiants pour le problème 2. Il est probable que ce problème soit plus facile que les deux autres, ce qui expliquerait ces différences de comportement.

Les analyses objective et subjective indiquent donc que c'est le processus d'analyse qui pose le plus de difficultés dans la résolution de problèmes. Cependant, ces résultats doivent être nuancés étant donné que le nombre de questions illustrant chacun des processus cognitifs n'est pas égal. La compréhension n'étant abordée que par une question, nous ne pouvons pas exclure que ce processus pose aussi des difficultés aux étudiants.

Des difficultés dans le processus d'analyse reflètent généralement une incapacité des étudiants à planifier correctement les tâches à réaliser pour résoudre le problème (Que dois-je mettre en œuvre ? Dans quel ordre dois-je

1 «Votre réponse vous parait-elle réaliste ?»; «Quelles sont les question(s) qui vous pose(nt) le plus de difficulté ?». 
le faire ?) et à réguler leurs actions (Comment réorienter mes actions en référence au feed-back fourni précédemment ?). En effet, le processus d'analyse implique une décomposition des différents éléments du problème afin de les simplifier pour pouvoir les mettre en relation et décider quelles actions appliquer et dans quel ordre. Ce processus implique aussi un ajustement des actions en cas d'échec de la procédure initiale. Des difficultés dans les questions, portant sur la détermination des informations utiles à la résolution du problème (question 5) et dans le chemin à adopter pour mettre en lien ces différents éléments (question 2), nous permettent d'énoncer que, de façon générale, les étudiants ont des difficultés à planifier leurs actions. Dans la partie résolution guidée du dispositif proposé, le processus d'analyse est simplifié par rapport à une démarche effectuée en dehors d'un tel dispositif. En effet, les étudiants ont des propositions de réponses sous les yeux; en aucun cas, ils ne doivent produire par euxmêmes des réponses (comme définir les informations utiles à la résolution du problème). Cette possibilité de choix parmi des réponses proposées devrait faciliter la production de réponses correctes chez les étudiants. Malgré ces conditions favorables à la mobilisation et à l'application du processus d'analyse, les étudiants restent en difficulté. Ces résultats tendent à montrer que ces derniers sont réellement en difficulté dans l'identification des éléments pertinents pour la résolution du problème et dans la définition des étapes à suivre pour le résoudre (planification).

L'identification du processus cognitif posant le plus de difficultés à l'étudiant (en l'occurrence généralement l'analyse) pourra lui être utile. En effet, en ayant conscience de ces difficultés, ils pourraient par eux-mêmes cibler leur travail et réaliser des activités entrainnant spécifiquement le ou les processus posant des difficultés. Cela donne la possibilité aux étudiants de s'investir dans leur propre remédiation. Comme le montre la méta-analyse de Means et al., les dispositifs en ligne permettant aux étudiants d'avoir une réflexion sur leur propre apprentissage et d'entrer en action pour remédier à des difficultés montrent de meilleurs résultats en termes d'apprentissage que ceux ne donnant pas aux étudiants ces possibilités (Means et al., 2010).

De la même manière, avoir facilement accès à ces données pourrait être utile aux enseignants dans le cadre d'une remédiation. En effet, sur la base de ces résultats, les enseignants pourraient mettre en place des activités permettant de travailler spécifiquement le processus posant le plus de difficultés, ici l'analyse.

Afin de faciliter l'utilisation de ces résultats comme outil réflexif pour les étudiants et comme base de remédiation, il est indispensable que chacun puisse visualiser rapidement les résultats. Pour cela, un outil concaténant l'ensemble des données issues de la résolution des problèmes sera mis au point. En quelques clics, l'enseignant pourra identifier quel étudiant est en difficulté dans quel(s) processus. Il appartiendra à l'enseignant d'utiliser ces données pour la réalisation d'activités ciblées. À son tour, en quelques clics, l'étudiant pourra savoir quel processus semble être le plus incertain chez lui. Nous sommes persuadés que son utilisation dans un cadre de remédiation sera favorable à l'apprentissage des étudiants.

\subsection{Y a-t-il un impact de l'entraînement en ligne sur la réussite à l'examen ?}

Si le dispositif aide les étudiants dans leur apprentissage, de meilleures performances à la résolution de problèmes proposés lors de l'examen (taux de réussite, note) devraient être constatées pour les étudiants ayant réalisé six problèmes en ligne par rapport aux étudiants n'ayant réalisé aucun problème en ligne.

Une description des résultats obtenus à l'examen et de la fréquentation du dispositif sera présentée, suivie d'une analyse de l'impact du travail en ligne sur la réussite et sur les performances pour les problèmes de résolution de l'examen de physique de juin 2012.

Sur 876 étudiants, $196(22,4 \%)$ ont réussi l'examen de physique en juin. Ce pourcentage est très faible et inférieur à celui obtenu dans les autres matières (autour de $50 \%$ ). Dans cette analyse, seuls les résultats obtenus aux différentes résolutions de problèmes seront considérés. Étant donné que le dispositif d'entraînement porte spécifiquement sur des problèmes de mécanique des fluides, les performances obtenues à la résolution du problème de mécanique des fluides seront isolées.

$10,84 \%$ des étudiants (soit 95 étudiants) ont réussi la partie résolution de problèmes passée lors de l'examen de juin (un problème de mécanique des fluides et un problème d'électricité). 7,08\% des étudiants (soit 62 étudiants) ont réussi le problème de mécanique des fluides proposé lors de l'examen de juin.

$\mathrm{Au}$ vu du faible taux de réussite pour les problèmes, un questionnement quant à la difficulté des problèmes proposés à l'examen peut être soulevé. Étant donné que les résultats aux examens sont encore plus faibles dans le cas du problème de fluide, la question de l'adéquation entre la difficulté des exercices proposés en cours et la difficulté du problème de l'examen peut être posée. Ce point sera abordé plus tard en conclusion.

Concernant le travail en ligne, sur 876 étudiants inscrits, 547 n'ont pas réalisé de problème (soit $62,5 \%$ ) et 156 ont réalisé les 6 problèmes (soit 17,8\%). 


\subsubsection{Performances des étudiants à l'examen de physique}

Voici un tableau récapitulatif des principaux effets :

\begin{tabular}{|l|l|l|l|}
\hline & $\begin{array}{l}\text { Test de } \\
\text { dépendance } \\
\mathrm{X}^{2} \text { de } \\
\text { Pearson }\end{array}$ & $\begin{array}{l}\text { Coefficients } \\
\text { d'association }\end{array}$ & Analyse des contributions \\
\hline $\begin{array}{l}\text { Dépendance entre la } \\
\text { réussite à la partie } \\
\text { résolution des problèmes } \\
\text { de l'examen et la } \\
\text { résolution de problèmes } \\
\text { en ligne }\end{array}$ & $\mathrm{P}<0.001$ & $\begin{array}{l}\text { Variation entre } \\
32,3 \% \text { et } \\
48,3 \%\end{array}$ & $\begin{array}{l}\text { La modalité contribuant } \\
\text { majoritairement à la dépendance } \\
\text { est « travail en ligne-réussite » }\end{array}$ \\
\hline $\begin{array}{l}\text { Dépendance entre la } \\
\text { réussite au problème de } \\
\text { mécanique des fluides de } \\
\text { l'examen et la résolution } \\
\text { de problème en ligne }\end{array}$ & $\mathrm{P}<0.001$ & $\begin{array}{l}\text { Force } \\
\text { d'association } \\
\text { faible à } \\
\text { moyenne }\end{array}$ & \\
& & $\begin{array}{l}\text { Variation entre } \\
28 \% \text { et 41,3 \% }\end{array}$ & $\begin{array}{l}\text { La modalité contribuant } \\
\text { Fajoritairement à la dépendance } \\
\text { d'association } \\
\text { faible à }\end{array}$ \\
moyenne & \\
\hline
\end{tabular}

\section{Tableau 5 : synthèse des effets de dépendance entre travail en ligne et réussite à la résolution de problèmes en physique}

Une dépendance entre travail en ligne et réussite aux problèmes présentés à l'examen de juin est constatée. Les forces d'association sont de faibles à moyennes.

Intéressons-nous maintenant aux éventuelles différences de notes obtenues par ces deux populations. Pour chaque groupe, les médianes des notes obtenues sur la partie résolution de problèmes de l'examen et le problème spécifique de mécanique des fluides ont été calculées. Si le travail en ligne aide effectivement les étudiants à résoudre des problèmes, les médianes des étudiants ayant travaillé en ligne devraient être supérieures à celles des étudiants n'ayant pas travaillé en ligne. Pour tester la significativité de ces potentielles différences, le test des médianes sera effectué.

Comme le montre la figure 2, lorsque les résultats obtenus sur la partie résolution de problèmes présentée à l'examen sont considérés, les étudiants ayant réalisé les 6 problèmes présentent une médiane significativement supérieure (médiane $=6$ ) à celle des étudiants n'ayant réalisé aucun problème en ligne (médiane $=0 ; \mathrm{p}<0,001$ au test des médianes).

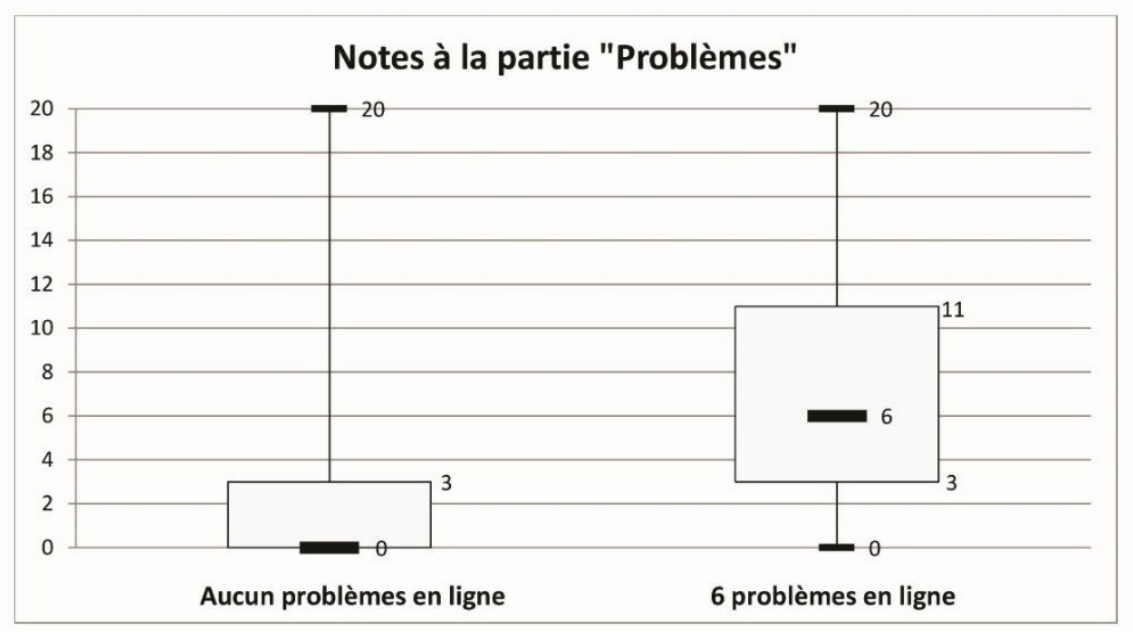

Figure $2:$ médianes des étudiants à

l'ensemble des problèmes présentés à l'examen en fonction du travail réalisé en ligne

En ce qui concerne les résultats obtenus pour le problème de mécanique des fluides présenté à l'examen, la tendance est exactement la même, bien que les notes soient plus basses. Comme le montre la figure 3 ci-dessous, la médiane 
des étudiants ayant réalisé 6 problèmes en ligne (médiane $=2$ ) est significativement supérieure à celle des étudiants n'ayant réalisé aucun problème en ligne (médiane $=0 ; \mathrm{p}<0,001$ au test des médianes).

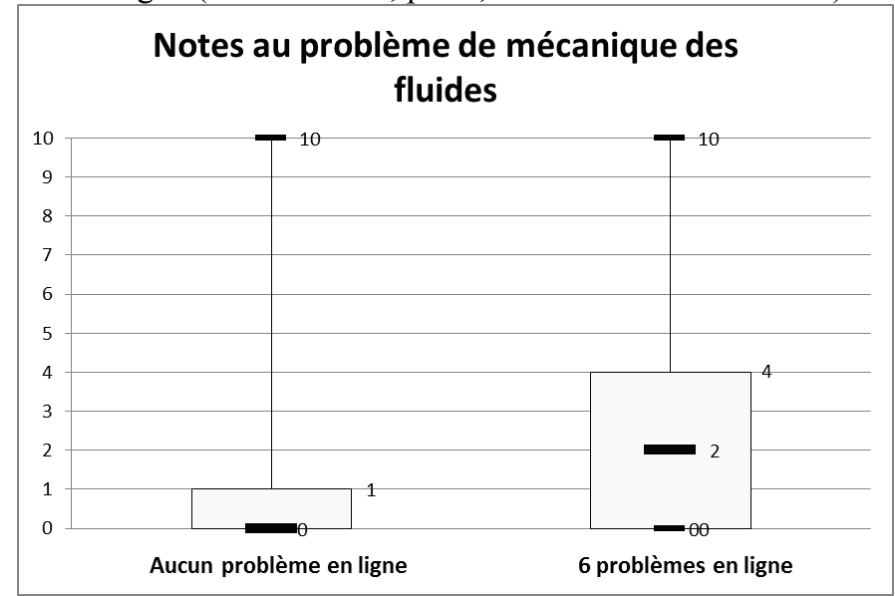

Figure 3 : médianes des étudiants au problème de mécanique des fluides présenté à l'examen en fonction du travail

$$
\text { réalisé en ligne }
$$

Il est donc clair que les étudiants ayant travaillé en ligne présentent, lors de la résolution de problèmes, des médianes plus élevées que ceux n'ayant pas travaillé en ligne.

Les différentes analyses effectuées mettent en évidence un lien entre travail en ligne et réussite ou performance à l'examen. Cependant, ces analyses permettent-elles d'affirmer que les performances des étudiants sont directement influencées par leur travail en ligne? N'est-il pas possible que les étudiants ayant réussi soient tout simplement des étudiants ayant des caractéristiques intrinsèques propices à la réussite universitaire (comme par exemple des méthodes de travail plus efficaces, une utilisation plus assidue des ressources mises à leur disposition, un niveau de départ plus élevé ou encore une capacité d'assimilation plus importante), et ce indépendamment de la qualité du dispositif en ligne proposé ? Il a souvent été observé que les dispositifs d'aide, comme les ressources mises à disposition par les enseignants, étaient majoritairement utilisés par les étudiants en ayant le moins besoin pour réussir, c'est-à-dire les étudiants les plus assidus, les moins en difficulté ou les plus engagés. Ce phénomène pourrait limiter l'impact du dispositif en mettant en valeur d'autres facteurs dans la réussite des étudiants.

\subsubsection{Performances des étudiants dans les autres matières scientifiques}

Afin de s'assurer que la réussite des étudiants est bien liée au travail en ligne et non à des caractéristiques intrinsèques des étudiants, les performances de ces étudiants dans les autres matières scientifiques vont être analysées. Travailler dans le dispositif proposé ne devrait pas avoir une influence sur les performances dans les autres matières que la physique. En effet, les processus impliqués dans la résolution de problèmes complexes ne sont pas à l'œuvre dans les examens de biologie ou de chimie. En biologie, l'examen comporte des questions à choix multiples portant essentiellement sur des notions théoriques. Aucune résolution de problèmes, ni d'analyse de données n'est demandée. En chimie, l'examen comporte des questions à choix multiples portant sur de la théorie et des applications de la théorie. Encore une fois, les processus de compréhension et d'analyse impliqués dans la résolution de problèmes complexes sont peu mobilisés. Si un lien entre travail en ligne et performances à l'examen des autres matières est mis en évidence, le rôle des caractéristiques propres à l'étudiant dans leur réussite (comme leur capacité d'assimilation, le niveau de départ ou encore leur engagement dans la tâche) devra être soulevé au détriment de celui du dispositif.

La question suivante sera donc posée dans un premier temps : les étudiants ayant réussi la partie résolution de problèmes de l'examen de physique ont-ils aussi réussi les autres matières scientifiques, à savoir la biologie et la chimie ? Si la réponse est positive, il sera impossible de conclure sur le rôle du dispositif d'aide dans la réussite des étudiants en résolution de problèmes de physique. En effet, ce dispositif n'a a priori pas les caractéristiques permettant d'affecter le travail en biologie ou en chimie. Si la réponse est négative (c'est-à-dire les étudiants ayant réussi la partie résolution de problèmes de physique n'ont pas systématiquement réussi les autres matières scientifiques), les performances des étudiants ayant réussi la résolution de problèmes en physique et travaillé en ligne seront particulièrement observées. Si ces étudiants ne montrent pas de capacité particulière dans les autres matières, le rôle du dispositif en ligne pourra être mis en avant dans la réussite de ces étudiants en physique. 
Dans un second temps, les notes obtenues seront analysées : y a-t-il une différence de médianes en biologie et en chimie entre les étudiants ayant travaillé en ligne et ceux ne l'ayant pas fait, et ce, que les étudiants aient réussi ou non chacun des deux examens? A priori, si le dispositif d'aide à la réussite permet d'améliorer la mobilisation et la réalisation des processus cognitifs impliqués dans la résolution de problèmes complexes, les médianes des étudiants dans les autres matières scientifiques ne devraient pas se distinguer en fonction de la réalisation de problèmes en ligne en physique. Une différence entre ces deux groupes d'étudiants viendrait appuyer le résultat précédent; en effet, cela tendrait à confirmer que la réussite des étudiants est due à d'autres facteurs que la participation au dispositif d'aide à la réussite.

Le premier point à noter est que les matières observées (biologie et chimie) sont beaucoup mieux réussies que la physique (taux de réussite de $52 \%$ ).

Voici un tableau récapitulatif des étudiants ayant réussi à la fois la résolution de problèmes en physique et une matière scientifique.

\begin{tabular}{|l|l|}
\hline & Nombre d'étudiants \\
\hline Étudiants ayant réussi les problèmes en physique & 95 \\
\hline Étudiants ayant réussi les problèmes en physique et à l'examen de biologie & 88 (soit 92,5\%) \\
\hline Étudiants ayant réussi les problèmes en physique et à l'examen de chimie & 87 (soit 91,5\%) \\
\hline
\end{tabular}

\section{Tableau 6 : nombre d'étudiants ayant réussi la résolution de problèmes en physique et en chimie et/ou biologie}

L'analyse montre clairement que les étudiants ayant réussi la résolution de problèmes en physique sont aussi ceux, dans une très large majorité, qui ont réussi les examens de biologie et de chimie. Il est probable que ces étudiants aient un meilleur niveau initial ou travaillent de façon plus efficiente, ce qui expliquerait leurs bonnes performances dans l'ensemble des matières scientifiques étudiées ici. Comme vu précédemment, les étudiants ayant réussi l'examen de physique ont majoritairement utilisé le dispositif en ligne mis à leur disposition; cependant à la vue de leur réussite conjointe dans les autres matières, conclure sur l'efficacité du dispositif dans la réussite des étudiants est délicat.

Voici un tableau récapitulatif des médianes obtenues dans chacune des matières scientifiques non entraînées par le dispositif d'aide à la réussite (biologie et chimie) et des analyses statistiques associées ${ }^{2}$.

\begin{tabular}{|l|l|l|l|}
\hline & $\begin{array}{l}\text { Médianes des étudiants } \\
\text { ayant travaillé en ligne }\end{array}$ & $\begin{array}{l}\text { Médianes des étudiants } \\
\text { n'ayant pas travaillé en } \\
\text { ligne }\end{array}$ & $\begin{array}{l}\text { Significativité au test } \\
\text { des médianes }\end{array}$ \\
\hline $\begin{array}{l}\text { Examen de } \\
\text { biologie }\end{array}$ & 12 & 9 & $\mathrm{P}<0,001$ \\
\hline $\begin{array}{l}\text { Examen de } \\
\text { chimie }\end{array}$ & 12 & 9 & $\mathrm{P}<0,001$ \\
\hline
\end{tabular}

\section{Tableau 7 : médianes des étudiants en chimie et biologie en fonction du travail en ligne et tests de significativité associés}

Comme le montre le tableau 7, les tests de la médiane appliqués respectivement aux résultats obtenus en biologie et en chimie mettent en évidence une différence entre les notes obtenues par les deux groupes. Les étudiants ayant travaillé en ligne présentent systématiquement une médiane supérieure à ceux n'ayant pas réalisé de problème sur la plateforme.

Ces données vont donc dans le même sens que celles considérant les pourcentages de réussite et les chevauchements entre matières : les performances des étudiants en résolution de problèmes en physique sont congruentes avec celles obtenues dans les autres matières. L'impact de l'utilisation du dispositif en ligne ne peut être clairement établi sur la base de la réussite à la résolution de problèmes en physique, ni sur la base de la qualité des notes obtenues dans cette matière. Il est probable que d'autres facteurs contribuent à cette réussite, et notamment des facteurs propres aux individus tels que la persévérance, l'assimilation ou encore le niveau de départ. Cependant, il ne peut non plus être établi que le dispositif n'a aucun impact. Malgré le fait que nous ne puissions pas explicitement conclure à un impact positif de ce dispositif sur la réussite des étudiants, le dispositif constitue une ressource supplémentaire que les étudiants pourront utiliser. Cependant, n'ayant pu organiser de prétest, il est difficile d'observer d'éventuelles évolutions de chaque étudiant liées à l'entrainement sur la plate-forme mise en place.

2 Chacun des examens dont il est question dans cette partie est noté sur 20 points. 


\section{Conclusion}

Le dispositif proposé est composé de deux méthodes principales de résolution de problèmes : la résolution guidée, basée sur les processus cognitifs de Bloom et d'Anderson ainsi que l'expérience des acteurs de terrain, et la résolution libre. Il permet aux étudiants d'être guidés de façon progressive à travers la résolution de problèmes. À chaque pas, un retour sur leur réalisation leur permet d'identifier leurs difficultés, de les comprendre et d'ajuster leurs actions en conséquence.

Étant donné la méthode de résolution guidée mise en place dans ce dispositif, il est aujourd'hui possible d'identifier le processus cognitif qui pose le plus de difficultés pour les étudiants dans la résolution de problèmes, à savoir le processus d'analyse. En analysant spécifiquement les questions, il apparaît que ce sont celles portant sur le chemin de résolution et la détermination des informations utiles qui sont les plus problématiques pour deux des trois problèmes proposés. Les étudiants semblent donc éprouver des difficultés à identifier ce qui pourrait leur être utile pour résoudre le problème, à planifier correctement les tâches à réaliser pour le résoudre et à réguler leurs actions. Pourtant, comme précisé précédemment, la méthode de résolution guidée les oriente pour une mobilisation efficace de ce processus d'analyse. En effet, ce processus est décomposé en questions auxquelles les étudiants doivent simplement répondre. Malgré ces conditions favorables à la mobilisation et à l'application du processus d'analyse, les étudiants restent en difficulté. Les analyses indiquent que de nombreux étudiants sont conscients de cette difficulté. Cette mise en évidence pourrait leur permettre de cibler leur travail et de réaliser des activités entrainant spécifiquement le processus d'analyse. Les enseignants ont été informés de ces résultats afin qu'ils puissent aider au mieux les étudiants en leur spécifiant des activités propices à la mobilisation et à une utilisation efficace du processus d'analyse.

Presque $20 \%$ des étudiants ont utilisé le dispositif de façon optimale (réalisation des six problèmes proposés) et volontaire. Les différentes analyses effectuées mettent en évidence que les étudiants ayant utilisé l'ensemble des ressources à disposition dans le dispositif ont de meilleurs résultats en résolution de problèmes à l'examen que les étudiants ne l'ayant pas fait. Cependant, les analyses indiquent aussi que les performances des étudiants en résolution de problèmes en physique sont congruentes avec celles obtenues dans les autres matières. Il est donc probable que les notes des étudiants ne soient pas uniquement influencées par le travail réalisé en ligne dans le dispositif. En effet, des caractéristiques propres aux individus telles que la persévérance, l'assimilation ou encore le niveau de départ interviennent dans leur réussite. Cependant, il ne peut non plus être établi que le dispositif n'a aucun impact ; il est une ressource supplémentaire proposée aux étudiants comme outil d'entrainement à la résolution de problèmes. L'absence de prétest ne permet pas d'observer une éventuelle évolution de chaque étudiant.

Ce dispositif permet aux enseignants d'envisager des pistes de remédiation et de proposer des activités directement liées aux besoins des étudiants. Cependant, son efficacité sur l'apprentissage n'est pas clairement établie. Un des éléments pouvant entraver l'efficacité d'un dispositif est le manque d'alignement pédagogique entre objectif, méthode et évaluation (Tyler, 1949). Une concordance entre ces trois piliers est-elle observable dans notre dispositif ? En revenant sur les objectifs du dispositif, il semble qu'ils aient été mal définis auprès des étudiants. Pour les concepteurs du dispositif, il est clair que l'objectif principal est de permettre aux étudiants d'appréhender la méthode de résolution de problèmes et d'augmenter leur compréhension de cette méthode et des concepts associés afin de leur permettre, notamment, de réussir leur examen. Selon Case et Marshall, cet objectif entre dans une approche de l'apprentissage de type "procedural deep approach»: « to gain understanding at some future point through familiarity with applications and problem-solving procedures $»^{3}$ (Case \& Marshall, 2004, p. 609).

Il est probable que l'objectif principal des étudiants soit tout simplement d'acquérir une méthode applicable lors de l'examen pour le réussir. La dimension compréhension profonde de la méthode n'est pas forcément incluse dans leurs intentions. Selon Case et Marshall, cet objectif entre dans une approche de l'apprentissage de type «procedural surface approach » (2004).

Les manques de définition et de communication autour des objectifs du dispositif donnent probablement lieu à une utilisation différente de celle attendue du dispositif. Le dispositif de résolution de problèmes pousse probablement les étudiants à croire qu'apprendre une méthode sans chercher à la comprendre de façon plus profonde est suffisant pour atteindre l'objectif qu'ils se sont fixé. Il semble donc important de clarifier les objectifs auprès des étudiants mais aussi de modifier la méthode en intégrant une dimension plus élaborée et profonde de l'apprentissage de la résolution de problèmes. Une façon de faire pourrait être d'envisager une intégration plus cohérente du dispositif dans l'enseignement. À l'heure actuelle, ce dispositif est utilisé comme une ressource complémentaire au cours. Étant donné qu'il a été identifié que le processus d'analyse est l'élément le plus difficile à mettre en place dans la résolution,

3 «to gain understanding at some future point through familiarity with applications and problem-solving procedures $»$. 
il serait important d'envisager une remédiation en présentiel autour du processus d'analyse et utilisant des problèmes en ligne.

Le troisième pilier, devant être concordant avec les objectifs et les méthodes pour qu'un dispositif permette un apprentissage de qualité, est l'évaluation. Les résultats à l'examen de physique sont très bas. Il est probable que l'ensemble de l'examen soit trop exigeant par rapport à la formation reçue par les étudiants. Cette situation est accentuée dans le cas de la résolution de problèmes, puisque moins de $11 \%$ des étudiants ont proposé une résolution correcte des deux problèmes proposés. La difficulté des problèmes proposés n'est semble-t-il pas en adéquation avec celle des problèmes proposés en ligne.

À l'avenir, l'accent sera mis sur une visualisation facile et rapide, tant pour les étudiants que pour l'équipe pédagogique, des données et progrès de chacun des étudiants. Un rapport pourra être généré et permettra à chacun d'identifier quel(s) processus cognitif(s), quelle(s) étape(s) de résolution posent des difficultés à l'étudiant. Une remédiation plus efficace pourra alors être mise en place puisqu'elle tiendra compte des difficultés spécifiques de chacun des étudiants. Cet outil d'entrainement et de remédiation permettra alors des étudiants d'aller à l'essentiel et de travailler concrètement et efficacement les matières et étapes de résolution de problèmes de physique lui posant le plus de difficultés.

\section{Bibliographie}

ANDERSON L.W., KRATHWOHL D.R., AIRASIAN P.W., CRUIKSHANK K.A., MAYER R.E., PINTRICH P.R., RATHS J. \& WITTROCK M. C. (2001). A taxonomy for learning, teacbing ans assessing: a revision of Bloom's taxonomy of eductional objectives. New York : Longman.

BANDURA A. (1980). L'apprentissage social. Bruxelles : Mardaga.

BLOOM B.S. (1956). Taxonomy of Educational Objectives, Handbook I: The cognitive Domain. New York : David McKay.

CASE J. \& MARSHALL D. (2004). Between deep and surface: procedural approaches to learning in engineering education contexts. Studies in Higher Education, vol. 29, n 5, p. 605-515.

JACQUET M., GEORGES F., GOURDANGE B., MICHIELS L., POUMAY M. (2012). En quoi un espace en ligne peut-il aider les étudiants de premier bachelier à résoudre des problèmes de physique ? Actes du colloque scientifique international portant sur les TIC en éducation : bilans, enjeux actuels et perspectives futures. Montréal.

LECLERCQ D., LASZLO P. \& DE LANDSHEERE G. (1978). Une expérience d'individualisation de l'enseignement de la chimie organique. Le Projet AMA. Bulletin de l'Université de Liège, nº 4, p. 22-23.

MEANS B., TOYAMA Y., MURPHY R., BAKIA M. \& JONES K. (2010). Evaluation of Evidence-based Practices in Online Learning: A Meta-analysis and Review of Online-learning Studies. Washington, D.C.: US Department of Education.

NEWELL A. \& SIMON H. A. (1972). Human problem solving. New Jersey: Prentice-Hall.

NICOL D. (2009a). Assessment for learner self-regulation: Enhancing achievement in the first year using learning technologies. Assessment and Evaluation in Higher Education, vol. 34, n 3, p. 335-352.

NICOL D. (2009b). Transforming assessment and feedback: enhancing integration and empowerment. En ligne : $<$ http://dera.ioe.ac.uk/11605/1/First_Year_Transforming_Assess.pdf $>$.

TARDIF J. (1992). Pour un enseignement stratégique. Montréal : Èd. Logiques-Écoles.

TYLER R. W. (1949). Basic principles of curriculum and instruction. Chicago : University of Chicago Press.

ZIMMERMAN B. J. (2002). Efficacité perçue et autorégulation des apprentissages durant les études: une vision cyclique. In P. Carré \& A. Moisan (éd.), La formation autodirigée, Paris : L'Harmattan, p. 69-88. 